\title{
Tomografía de impedancia eléctrica en la lesión pulmonar aguda
}

\author{
J. Rieraa ${ }^{a, *}$, P.J. Riu ${ }^{b}$, P. Casan ${ }^{c}$ y J.R. Masclans ${ }^{a}$ \\ a Servicio de Medicina Intensiva, Hospital Universitario Vall d'Hebron, Vall d'Hebron Institut de Recerca, Universitat Autònoma \\ de Barcelona, Barcelona, España \\ ${ }^{\mathrm{b}}$ Centre de Recerca en Enginyeria Biomedica, Departament d'Enginyeria Electronica, Universitat Politecnica de Catalunya, \\ Barcelona, España \\ c Servicio de Neumología, Hospital Universitario Central de Asturias, Oviedo, Facultad de Medicina y Ciencias de la Salud de la \\ Universidad de Oviedo, Oviedo, España
}

Recibido el 29 de marzo de 2011; aceptado el 3 de mayo de 2011

Disponible en Internet el 15 de junio de 2011

\section{PALABRAS CLAVE \\ Tomografía de impedancia eléctrica; Impedancia; Lesión pulmonar aguda; Monitorización pulmonar no invasiva}

\section{KEYWORDS}

Electrical impedance tomography; Impedance; Acute lung injury; Non-invasive pulmonary monitoring

\begin{abstract}
Resumen La tomografía de impedancia eléctrica se ha descrito como un nuevo método de monitorización en el paciente crítico en ventilación mecánica. Recientemente ha cobrado especial interés, debido a su aplicabilidad para la monitorización de la ventilación y la perfusión pulmonar. Su implementación continua a pie de cama y el ser una técnica no ionizante y no invasiva son propiedades particulares que la convierten en un recurso extremadamente atractivo. Asimismo, por su capacidad de evaluar las características regionales de la estructura pulmonar, podría constituir una herramienta de monitorización ideal en el heterogéneo pulmón con lesión pulmonar aguda.

En el presente artículo de revisión, se explica el concepto físico de la bioimpedancia y su aplicación clínica y se resume la evidencia científica publicada hasta la fecha en lo referido a la utilización de la tomografía de impedancia eléctrica como método de monitorización de la ventilación y de la perfusión, fundamentalmente en el enfermo con lesión pulmonar aguda, así como otras aplicaciones posibles de la técnica en el enfermo crítico. Asimismo, se resumen las limitaciones de la técnica y sus potenciales áreas de desarrollo en el futuro.

(c) 2011 Elsevier España, S.L. y SEMICYUC. Todos los derechos reservados.
\end{abstract}

\section{Electrical impedance tomography in acute lung injury}

Abstract Electrical impedance tomography has been described as a new method of monitoring critically ill patients on mechanical ventilation. It has recently gained special interest because of its applicability for monitoring ventilation and pulmonary perfusion. Its bedside and continuous implementation, and the fact that it is a non-ionizing and non-invasive technique, makes it an extremely attractive measurement tool. Likewise, given its ability to assess the regional characteristics of lung structure, it could be considered an ideal monitoring tool in the heterogeneous lung with acute lung injury.

\footnotetext{
* Autor para correspondencia.

Correos electrónicos: jorriera@vhebron.net, jordialg@hotmail.com (J. Riera).
} 
This review explains the physical concept of bioimpedance and its clinical application, and summarizes the scientific evidence published to date with regard to the implementation of electrical impedance tomography as a method for monitoring ventilation and perfusion, mainly in the patient with acute lung injury, and other possible applications of the technique in the critically ill patient. The review also summarizes the limitations of the technique and its potential areas of future development.

(C) 2011 Elsevier España, S.L. and SEMICYUC. All rights reserved.

No se puede pisar dos veces en el mismo río, porque las aguas nuevas siempre están fluyendo encima de ti. En el Teetetes de Platón.

\section{Introducción}

La tomografía de impedancia eléctrica (TIE) utiliza el principio físico de la impedancia para evaluar diferentes propiedades tisulares. Se trata de una herramienta de diagnóstico que utiliza las características eléctricas del tejido para dar información de manera no invasiva, continua, a pie de cama y sin radiación. En el pulmón ventilado con lesión pulmonar aguda (LPA) la técnica tiene particular interés al tener la capacidad de proporcionar datos importantes de lo acontecido en un corte axial del parénquima pulmonar que, por definición, es un tejido heterogéneo con zonas con diferentes características mecánicas. Esto provoca que los parámetros globales (de presión y volumen fundamentalmente) que ofrece el ventilador, tengan distinta repercusión en diferentes áreas, pudiendo combinarse unidades alveolares sobredistendidas con unidades alveolares colapsadas ${ }^{1}$ y estas áreas pueden tener, además, un diferente grado de perfusión tisular. La capacidad de la TIE para mostrar las características regionales en cuanto a ventilación y perfusión se ha ido perfeccionando a lo largo de estos últimos años, considerándose actualmente una técnica que podría ser de ayuda a la hora de optimizar los parámetros pautados en el ventilador. Por otro lado, puede aportar otra información importante en el enfermo crítico como es la medición del gasto cardíaco, la localización de una ocupación pleural o la confirmación del correcto emplazamiento del tubo orotraqueal. No obstante, deben conocerse las limitaciones del método, fundamentalmente asociadas a la técnica de calibración, y hay que saber interpretar la información ofrecida por el tomógrafo en relación con los cambios clínicos acontecidos en el enfermo.

\section{El principio físico y su aplicación}

La impedancia es una variable física que describe las características de la resistencia de un circuito eléctrico en la presencia de una corriente alterna. Es la oposición total al paso de corriente. Matemáticamente es un número complejo formado por una parte real (la resistencia) y otra imaginaria (reactancia). La unidad de impedancia es el ohm $(\Omega)$.

Si aplicamos esta variable a un tejido biológico, hablamos entonces de bioimpedancia. Diferentes tejidos biológicos tienen distinta resistencia al paso de corriente (tabla 1). No obstante las cifras no son absolutas y pueden variar con las condiciones del medio, como por ejemplo con la tem- peratura. Un aumento de la temperatura produciría una disminución en la impedancia, debido a cambios en la movilidad de los iones. Los tejidos se pueden modelar utilizando un circuito eléctrico de dos terminales, cuya impedancia represente a la del tejido estudiado ${ }^{2,3}$.

Utilizando múltiples electrodos es posible obtener imágenes de la bioimpedancia de una sección del cuerpo, lo que se denomina TIE. El principio de la TIE está basado en las medidas repetidas de los voltajes de superficie, resultantes de una inyección rotatoria de corriente alterna de baja intensidad entre electrodos situados en una circunferencia que rodea el objeto estudiado ${ }^{4}$.

El hardware utilizado para la TIE ha sido extensamente analizado en la literatura ${ }^{5,6}$. Simplificando su estructura, el sistema consiste en un sistema de inyección de corriente y detección de tensión multicanal, una computadora y 16 electrodos (opcionalmente se pueden aplicar 1 o 2 neutrales) aplicados al tórax del paciente (fig. 1). El número de electrodos puede ser variable, aumentando la resolución de las imágenes cuantos más electrodos se utilicen. La región torácica de aplicación de los electrodos es de vital importancia dada la potencial interferencia de otras estructuras como el corazón y el diafragma en la obtención de imágenes pulmonares. Normalmente se aplican a nivel del sexto espacio intercostal. Estos electrodos recogen la información de la impedancia en un espacio cráneo-caudal de aproximadamente $10 \mathrm{~cm}^{5}$. Generalmente las mediciones se basan en la aplicación de una corriente alterna de $50-80 \mathrm{kHz}$ (el uso de más de una frecuencia es posible, pero raramente utilizada en la monitorización de la impedancia pulmonar) y de baja intensidad (5 mApp) entre dos electrodos contiguos. El resto de pares de electrodos detectan el voltaje de la señal eléctrica que dependerá de las características del tejido que haya atravesado. Esta información es registrada por la computadora. Inmediatamente después, el siguiente

Tabla 1 Resistividad de diferentes tejidos (frecuencia de $50 \mathrm{kHz}$ )

\begin{tabular}{ll}
\hline Tejido & Resistividad $(\Omega \mathrm{m})$ \\
\hline Pulmón (espiración) & 12,5 \\
Pulmón (inspiración) & 25 \\
Sangre (Hc 50\%) & $1,4-1,7$ \\
Músculo cardíaco & 2,5 (longitudinal), 5 (transversal) \\
Músculo esquelético & 1,5 (longitudinal), 5 (transversal) \\
Hígado & 8,3 \\
Grasa & $10-50$ \\
Hueso & 160 (radial) \\
\hline
\end{tabular}




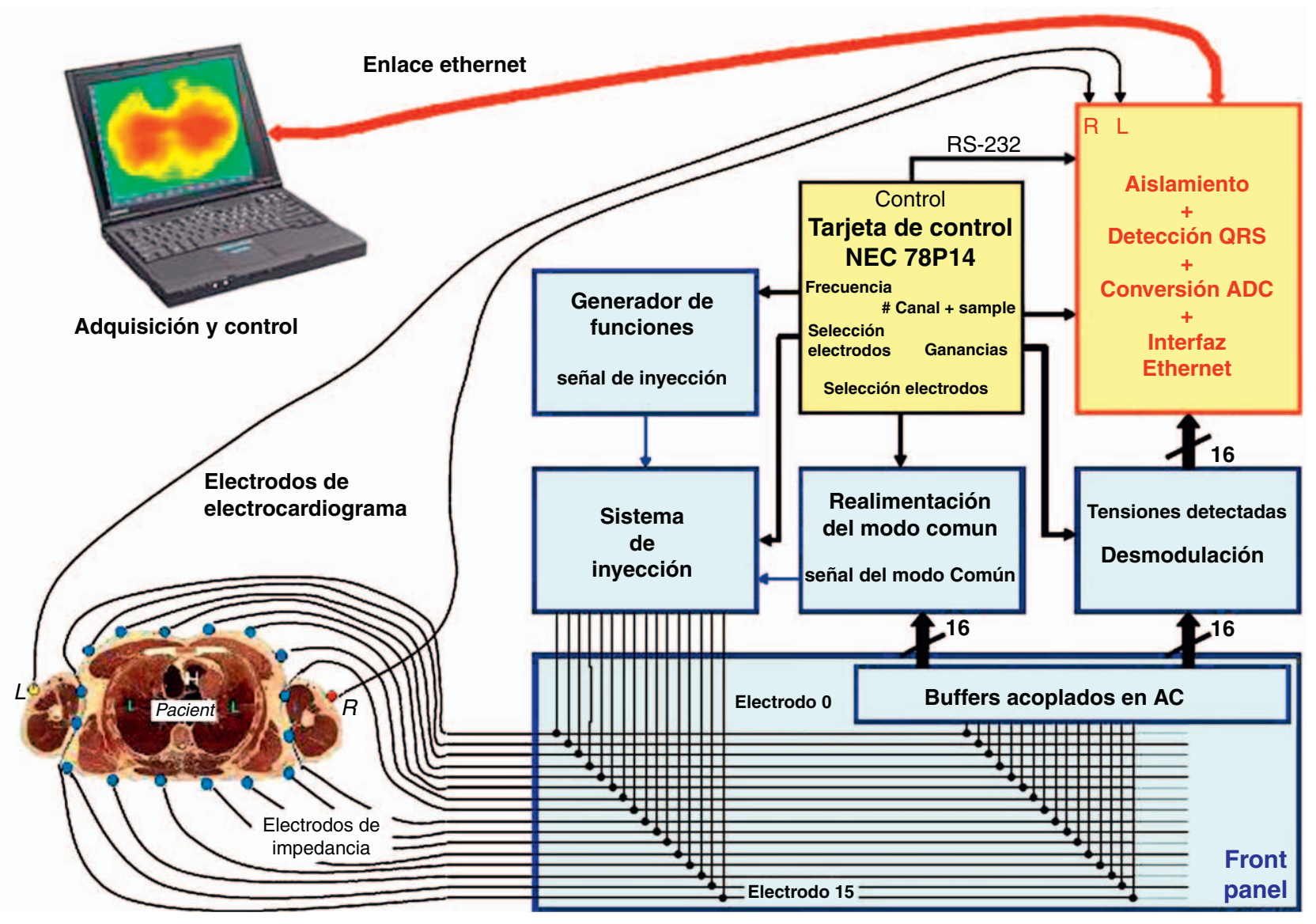

Figura 1 Ejemplo esquemático de hardware de tomografía de impedancia eléctrica. (Fuente: M. Balleza).

par de electrodos inyecta la corriente, recibiendo el voltaje resultante el resto de pares de electrodos. Cada vuelta completa de la medida de la impedancia de la sección del tórax se denomina ciclo. Los tomógrafos actuales completan por lo general 25 ciclos por segundo. Con 16 electrodos, se puede generar una imagen del plano definida por una matriz de 1.024 píxeles, aunque la resolución real con 16 electrodos es de aproximadamente el 1\% del área, y no es uniforme en toda la sección. Es de importancia crucial el método empleado para la reconstrucción de la información en imágenes ${ }^{7,8}$. Se han discutido diferentes métodos de reconstrucción y algoritmos en la literatura ${ }^{9-12}$. El más empleado en aplicaciones clínicas es el Sheffield backprojection algorithm y sus modificaciones posteriores ${ }^{5,13,14}$. Estas reconstrucciones asumen una forma redondeada del corte axial del tórax, por lo que introducen información $a$ priori en la representación de los datos.

Las medidas de bioimpedancia pueden ser clasificadas en dos tipos. El primero implica la determinación de las características de los tejidos corporales tales como el grado de edema o la cantidad de grasa. Un aumento del contenido de agua extracelular, una alta concentración de electrolitos y un número elevado de uniones celulares reducen la impedancia. La grasa, el hueso y el aire actúan como elementos resistores, incrementando, así, la impedancia regional. Este tipo de medición se aplica, fundamentalmente, en nutrición y en medicina del deporte. El segundo es el estudio de los cambios de impedancia asociados, principalmente, con el sistema respiratorio y circulatorio. Este tipo de estudio es el que emplea la TIE funcional $\left(\mathrm{TIE}_{\mathrm{f}}\right)$. Para la reconstrucción de la información, la $\mathrm{TIE}_{f}$ emplea el cambio de la impedancia relativa en cada píxel. Este valor (adimensional) deriva de la diferencia de la impedancia del tejido entre dos instantes en el tiempo (fig. 2). La reconstrucción de la distribución de la impedancia absoluta requiere el conocimiento de la forma del corte axial del tórax ${ }^{14}$. Esto es extremadamente difícil, ya que la sección no tiene una forma homogénea. Así, se emplea la reconstrucción basada en los cambios de la impedancia con relación a una referencia ya que se asume que la forma del tórax no cambia entre las mismas. Esta interpretación minimiza los errores derivados de asumir una forma incorrecta de la sección del tórax y su validez ha sido demostrada en los últimos años. Por tanto, la medida del cambio de la impedancia relativa permite la comparación de dos condiciones fisiológicas diferentes (por ejemplo, antes y después de cambiar los parámetros del ventilador o el cambio entre inspiración y espiración).

El cambio en la bioimpedancia torácica se ve influido fundamentalmente por dos mecanismos cíclicos: la ventilación y la perfusión. El incremento en la cantidad de aire durante la inspiración, junto con el incremento de volumen del pulmón y el cambio de volumen de la caja torácica, conduce a un aumento de la impedancia que es proporcional al volumen de gas inspirado, aunque la constante de proporcionalidad depende de cada sujeto. Por otro lado, la perfusión pulmonar provoca cambios pequeños, del orden del $3 \%$, en la impedancia torácica entre sístole y diástole. 
Tie en inspiración

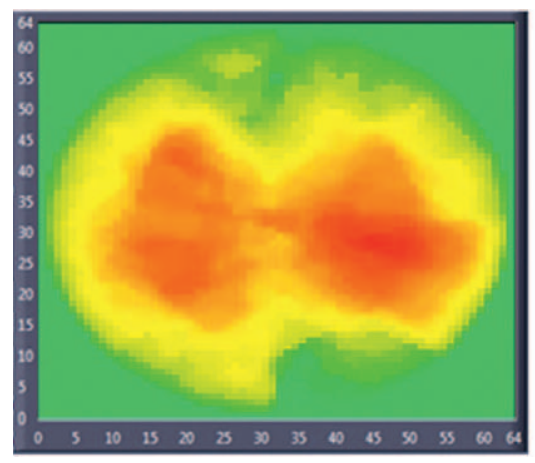

Individuo sano

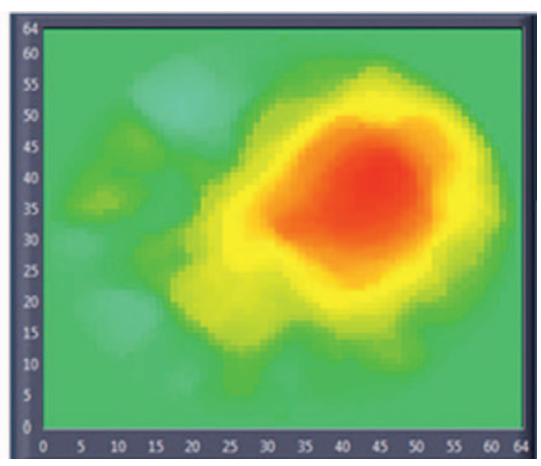

Individuo neumectomizado

Tie en espiracion

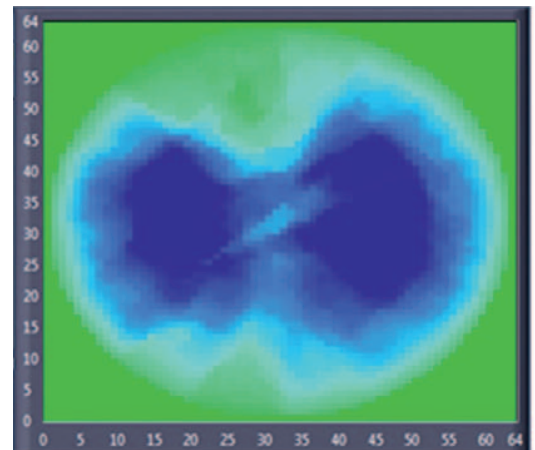

Individuo sano

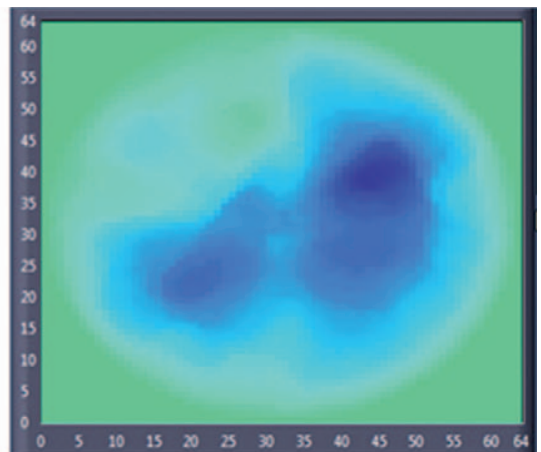

Individuo neumectomizado

Variabilidad

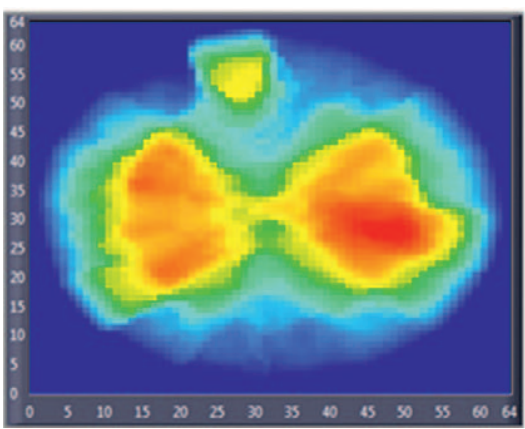

Individuo sano

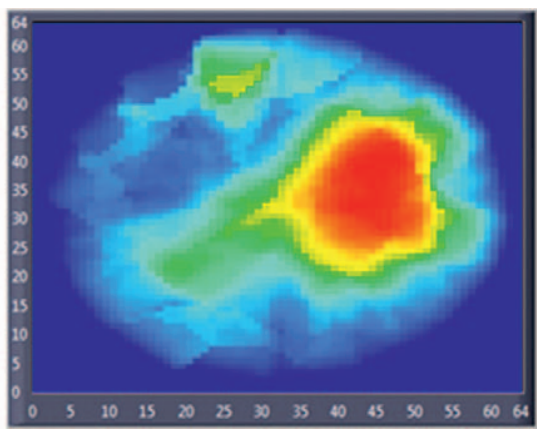

Individuo neumectomizado

Figura 2 Imágenes de tomografía de impedancia eléctrica en individuo sano e individuo neumectomizado. En inspiración, en espiración e imágenes de variabilidad. Las imágenes de inspiración y espiración muestran la variación de impedancia respecto a una referencia. Las imágenes de variabilidad corresponden a la desviación estándar de cada píxel en un lapso de tiempo (30 segundos).

\section{Monitorización de la ventilación alveolar}

El tejido pulmonar tiene una resistencia al paso de corriente que es cinco veces mayor al resto de tejidos blandos intratorácicos. Durante el proceso cíclico de la respiración la impedancia del tejido pulmonar cambia un $5 \%$ en respiración tranquila y hasta un $300 \%$ si se realiza una maniobra de inspiración desde el volumen residual hasta el volumen de capacidad total pulmonar ${ }^{15}$, mientras que la impedancia de la pared torácica permanece relativamente constante. Creando una imagen de los cambios en la distribución de la impedancia, se puede objetivar la distribución de la ventilación.

El análisis de los cambios de la impedancia relativa en un área de interés particular (RI) puede ser empleado para calcular la amplitud del cambio de bioimpedancia (volumen corriente) ${ }^{16,17}$, la impedancia mínima (capacidad residual funcional) ${ }^{18}$ o máxima (capacidad pulmonar 
total) ${ }^{19}$. También se ha utilizado para observar la distribución del volumen de gas en el pulmón variando los parámetros del ventilador.

Se han publicado numerosos estudios de validación de la TIE. Hasta la fecha, ningún estudio ha demostrado resultados negativos. Ya en el año $1995 \mathrm{Hahn}$ et al compararon la técnica con la espirometría para la validación de los cambios en la impedancia regional y global ${ }^{20}$. Más recientemente, Hinz et al la compararon con el método de lavado de nitrógeno para la medición del volumen pulmonar al final de la espiración (EELV) en pacientes ventilados con $\mathrm{LPA}^{21}$. Con el mismo fin, Marquis et al utilizaron la pletismografía ${ }^{22}$. En cuanto a su validación como método para la medición de distribución regional del volumen pulmonar, en varios estudios se han utilizado técnicas como la tomografía computarizada (TC) y la tomografía de emisión de positrones (SPECT y PET). Wrigge et al aplicaron la TIE para monitorizar el reclutamiento en una maniobra de inflado pulmonar lento en un modelo experimental de LPA $^{23}$. Asimismo, Frerichs et al compararon la técnica con la TC para detectar cambios regionales en el volumen pulmonar resultantes de cambios en los parámetros del ventilador en modelos animales con pulmón sano, viendo buena correlación entre ambas mediciones ${ }^{24}$. También Victorino et al aplicaron la TC y la TIE para medir la influencia de la gravedad en la ventilación regional, encontrando una alta correlación entre las dos técnicas ${ }^{25}$. Por otro lado, Hinz et al validaron la utilización de la técnica para la medición de la ventilación regional, comparando los datos con la SPECT en un modelo animal de $\mathrm{LPA}^{26}$. Recientemente, Richard et al compararon la TIE con la PET para cuantificar los cambios en la ventilación regional consecuentes a cambios en los parámetros del ventilador en modelos animales de pulmón sano y con LPA, observando que tanto en una población como en otra existía una excelente correlación entre las mediciones obtenidas mediante una técnica y otra ${ }^{27}$. Los datos derivados de todos estos estudios sugieren que los cambios en la impedancia relativa medidos por la TIE pueden ser empleados para la cuantificación de la ventilación regional con suficiente precisión.

Se ha observado que los parámetros globales, como las curvas presión-volumen o la medición de la compliance del sistema respiratorio, no traducen fehacientemente lo que en realidad está ocurriendo en la estructura de un pulmón en situación de LPA. La intrínseca heterogeneidad de la afección, con la coexistencia de unidades alveolares colapsadas y otras sobredistendidas, distorsiona estas mediciones. En este sentido, la TIE ofrece ventajas importantes fundamentalmente debido a la posibilidad que ofrece de análisis de la distribución regional del volumen pulmonar. Es de esencial importancia encontrar una presión espiratoria positiva (PEEP) adecuada en la estrategia de ventilación, especialmente en el pulmón con LPA. Múltiples estudios han sido publicados en los que se intenta determinar mediante TIE la PEEP óptima, que permita un mínimo desreclutamiento y una mínima sobredistensión ${ }^{28-34}$. Kunst et al utilizaron la técnica para calcular los puntos de inflexión superior e inferior de la curva presión (global)-volumen (regional) en diferentes RI en modelos animales con LPA ${ }^{35}$. Así lo hicieron también Hinz et al en pacientes ventilados, concluyendo que los puntos de inflexión de la curva presión-volumen obtenidos mediante parámetros globales no eran representativos de todas las áreas del pulmón ${ }^{36}$. Asimismo, Lowhagen et al utilizaron la TIE para la identificación del punto inferior de inflexión de la curva de presión-volumen en las regiones dorsales en 16 enfermos ventilados con LPA ${ }^{37}$. Kunst et al publicaron también un estudio donde monitorizaban el reclutamiento y el desreclutamiento pulmonar con diferentes protocolos ventilatorios en un modelo animal de LPA ${ }^{38}$, concluyendo que la técnica podría ser un excelente método para la elección de los parámetros óptimos del ventilador. Lowhagen et al observaron, tras diferentes cambios en los parámetros del ventilador, los cambios regionales en el EELV y la distribución del volumen corriente en el pulmón ${ }^{39}$. También se ha empleado la TIE como método de monitorización en modos no convencionales de ventilación mecánica como en ventilación de alta frecuencia (VAFO). Van Genderingen et al midieron, en un modelo animal de LPA, la distribución del volumen pulmonar con TIE durante una maniobra de inflado, viendo que la distribución no era homogénea, y mediante la aplicación de VAFO, comprobando en ese caso que la distribución era más homogénea ${ }^{40}$.

Por lo tanto, considerando todos estos estudios, se demuestra que la TIE es un interesante método de monitorización durante la maniobra de ventilación mecánica, por su capacidad de lectura regional y su no invasividad. Otra ventaja que ofrece el sistema es su continua lectura de las condiciones cambiantes del pulmón ventilado, pudiendo identificar, a pie de cama, cambios en la mecánica pulmonar secundarios a maniobras frecuentes como puede ser la despresurización del sistema tras una maniobra de aspiración de secreciones ${ }^{41}$, o los cambios en la ventilación regional después de una modificación posicional del enfermo tras una movilización.

\section{Monitorización de la perfusión pulmonar}

Se ha confirmado que, además de los cambios en la impedancia relativos al proceso de movimiento de volumen de aire, se pueden objetivar los cambios en la impedancia relacionados con la perfusión del tejido pulmonar. Al perfundirse los pulmones, ocurre una caída de la impedancia en un porcentaje variable $e^{42,43}$. Dada la amplia magnitud del componente ventilatorio del cambio en la impedancia torácica, es importante poder aislar los dos procesos. Se pueden diferenciar los cambios en la impedancia relativos a la perfusión de los cambios relativos a la ventilación mediante varias maniobras. Fagerberg et al emplearon una pausa espiratoria para evaluar los cambios relativos a la perfusión pulmonar en 6 modelos porcinos en ventilación mecánica ${ }^{44}$. Deibele et al describen en su estudio un método dinámico de filtro para aislar los cambios de impedancia relativos a la perfusión en dos individuos sanos en respiración espontánea con aceptables resultados, aunque se concluye que su validez ha de ser confirmada en estudios ulteriores ${ }^{45}$. También se ha empleado una inyección de suero salino hipertónico como un agente de contraste en el intento de lograr una independencia de los cambios relativos a la perfusión ${ }^{46}$.

McArdle et al, ya en el año 1988, encontraron correlación entre las imágenes obtenidas mediante TIE y las imágenes conseguidas con TC tras inyección de isótopo por perfusión con el fin de analizar la perfusión pulmonar en tres pacientes, uno con embolismo pulmonar, otro con una 
bulla enfisematosa y otro con ausencia de perfusión del lóbulo inferior izquierdo ${ }^{47}$. Smit et al estudiaron la perfusión pulmonar con TIE en 24 individuos sanos observando que los resultados eran reproducibles al ser analizados por dos investigadores diferentes ${ }^{48}$. El mismo grupo estudió el efecto de la hiperoxia y la hipoxia en la vasoconstricción pulmonar en 7 voluntarios sanos y seis pacientes con enfermedad pulmonar obstructiva crónica (EPOC) empleando la $\mathrm{TIE}^{49}$. Se ha demostrado la potencial aplicación de la TIE en la identificación de embolismos pulmonares con amplia afectación pulmonar, aunque no en la identificación de los embolismos pequeños ${ }^{50}$. Sin embargo, solo un estudio de validación se ha publicado hasta la fecha comparando los hallazgos de la TIE relativos a la perfusión pulmonar con una prueba gold standard ${ }^{51}$.

Sin duda, esta capacidad de medida de la perfusión pulmonar regional, es de sumo interés en la monitorización de numerosas afecciones. Una de ellas es la LPA. La identificación de las áreas mejor perfundidas puede ayudar a ajustar de manera más eficiente los parámetros del ventilador en busca del reclutamiento del espacio aéreo en estas regiones, hecho que, como ya se ha explicado, la misma técnica ayuda a llevar a cabo. Esto podría optimizar la relación ventilación/perfusión ${ }^{44,52}$. No obstante, se requiere desarrollar la metodología en la interpretación de la señal relativa exclusivamente a los cambios en la perfusión pulmonar y la identificación de los papeles que juegan en dichos cambios el volumen sistólico del ventrículo derecho, los grandes vasos y los capilares pulmonares.

\section{Otras aplicaciones}

Existen otras aplicaciones interesantes de la TIE en el enfermo crítico. Se ha empleado para monitorizar el grado de edema pulmonar ${ }^{53}$. En este sentido, un área de extraordinario interés a explorar es el de la identificación de algún factor característico que pudiera diferenciar el edema producido por una mera sobrecarga hidrostática del asociado a procesos inflamatorios. También la TIE se ha empleado para detectar la ocupación pleural por aire o por líquido ${ }^{54,55}$. La técnica puede llegar a identificar volúmenes de aire tan bajos como de $20 \mathrm{ml}$. Esta posibilidad es extremadamente atractiva en el paciente crítico ventilado con tendencia a desarrollar derrame pleural y en riesgo de sufrir barotrauma asociado a la ventilación (fundamentalmente si existe LPA). Los cambios inmediatos en la bioimpedancia asociados a estos hallazgos ofrecen la posibilidad de iniciar medidas terapéuticas precoces al ser este un modo de monitorización continuo. Asimismo se ha publicado un estudio donde se emplea la TIE para confirmar el correcto emplazamiento del tubo orotraqueal ${ }^{56}$, concretamente en 40 pacientes con ventilación diferencial. La técnica podría traducir también la correcta colocación del tubo orotraqueal convencional durante la intubación y durante todo el proceso de ventilación mecánica, pudiendo identificarse potenciales intubaciones selectivas de una manera precoz. Otras aplicaciones que ofrece la técnica son la monitorización de la función pulmonar unilateral ${ }^{57}$ y la cuantificación del volumen sistólico derecho ${ }^{58}$, siendo comparada la técnica de cardiografía de impedancia con la termodilución con catéter de arteria pulmonar (CAP) encontrando una buena correlación e incluso mayor reproducibilidad en un mismo paciente ${ }^{59}$. Todo ello asociado a una lógica menor invasividad de la TIE en comparación al CAP.

La TIE presenta gran número de potenciales aplicaciones en la investigación de la fisiología pulmonar, tal como se observa en las publicaciones de Lindley et al y Frerichs et al en las que se estudian los efectos de la gravedad en la distribución del volumen pulmonar en individuos sanos durante vuelos parabólicos ${ }^{60}$.

\section{Limitaciones de la técnica}

Con la TIE, obviamente, solo se obtienen imágenes de impedancia en un corte axial del tórax, sin tener en cuenta el resto del parénquima pulmonar. Por otra parte, la resolución espacial de la técnica, tanto en lo referente a la ventilación (cada píxel contiene la información de la impedancia de varias unidades alveolares) como a la perfusión, es todavía baja. En este sentido, hay que tener claro que la TIE ofrece imágenes de función y no imágenes anatómicas. Sería posible mejorar la resolución espacial de la técnica pero es improbable que alcance un nivel de definición comparable a la TC o la resonancia magnética.

Como ya se comentó, es un punto crucial el hecho de que la calibración permita una traducción exacta de los cambios de impedancia en imágenes y estos en cambios de volumen de manera mantenida en el tiempo. Esta calibración no es sencilla y puede asumir errores que resten fiabilidad al sistema de monitorización. Dicho aspecto ya complicó el desarrollo de la pletismografía inductiva como método no invasivo de monitorización de la ventilación ${ }^{61}$.

Las imágenes de TIE traducen cambios en la impedancia del tejido pulmonar, no valores absolutos. Por lo tanto, condiciones existentes previamente a la monitorización con este sistema (por ejemplo áreas consolidadas, derrame pleural o bullas aéreas) no están representadas en las mismas.

Los potenciales cambios en la geometría torácica entre las diferentes maniobras pueden distorsionar las mediciones realizadas, por lo que se asume este potencial error. Además de los cambios en la geometría torácica, otros factores pueden alterar la medida en una de las características particulares. Por ejemplo, al medir los cambios en la ventilación, se pueden estar superponiendo los cambios en los fluidos intratorácicos (derrame pleural, agua pulmonar extravascular, volumen sanguíneo pulmonar) que pueden variar, asimismo, la medida de impedancia ${ }^{62}$. Es fundamental, por tanto, asociar los cambios objetivados por el sistema con los cambios clínicos del enfermo, hecho que no es exclusivo de esta técnica de monitorización, para una correcta interpretación de los mismos.

La comparación interindividual e incluso intraindividual de los valores de impedancia, especialmente entre periodos largos de tiempo y tras varios cambios en las condiciones pulmonares, es un proceso complicado. La correlación de los cambios de la impedancia con los de volumen puede no ser del todo exacta como demuestran Bikker et al en su reciente artículo en el que, en 25 pacientes ventilados, con diferentes niveles de PEEP intentaron correlacionar el EELV con la impedancia al final de la espiración (EELI) concluyendo que la relación lineal que existe en respiración normal entre cambios de volumen y cambios de impedancia no se 
puede usar para determinar EELV (que es un valor absoluto, no un cambio) ${ }^{63}$. Estas limitaciones se podrían minimizar, en parte, consiguiendo adecuadas técnicas de normalización y de calibración.

\section{Nuevas áreas de investigación}

El desarrollo de nuevo hardware y software que permitan una mayor resolución espacial optimizaría los resultados de las mediciones, tanto en lo relativo a la ventilación como a la perfusión. Asimismo, se debería desarrollar la técnica para poder obtener una distribución espacial de las impedancias absolutas con más precisión y, así, no depender de mediciones relativas que pueden pasar por alto condiciones preexistentes, como se explicó con anterioridad.

También ya se ha comentado que la TIE por el momento solo da información de un corte axial del tórax. El desarrollo de un sistema capaz de crear una imagen en tres dimensiones, mejoraría la capacidad de lectura del sistema.

Con el desarrollo de la técnica se podría pasar de identificar cambios groseros en las estructuras estudiadas a independizar los hallazgos identificando particularidades específicas de los mismos como puede ser la diferenciación entre la aparición de edema hidrostático del edema generado por inflamación. En esta misma línea, también se podría ofrecer la TIE como otro método de medida por imagen de la evolución de una neumonía e incluso suponer una herramienta de apoyo en el algoritmo de su diagnóstico.

Por último, la técnica podría ser utilizada como método de estudio de los efectos en el pulmón con LPA de otras maniobras asociadas al tratamiento de la hipoxemia refractaria como son el decúbito prono o el óxido nítrico inhalado.

\section{Conclusiones}

En el actual contexto de toma de conciencia de que las habituales maniobras terapéuticas poseen intrínsecamente potencial para aumentar el daño ya presente en el enfermo crítico, la monitorización de los efectos de las mismas sobre el organismo adquiere una importancia fundamental. La acertadísima sentencia Less is more implica una capacidad para medir. La TIE ofrece ventajas evidentes a la hora de observar lo que las maniobras terapéuticas aplicadas, fundamentalmente relacionadas con la VM, provocan en el pulmón afecto de LPA. La capacidad de lectura regional, su aplicabilidad a pie de cama y el ser una técnica segura, permiten intuir que la TIE tendrá cada vez un papel más importante a la hora de permitir minimizar los efectos indeseables de nuestras maniobras, como es el daño intrínseco de la ventilación con presión positiva sobre el pulmón enfermo.

\section{Conflicto de intereses}

Los autores declaran no tener ningún conflicto de intereses.

\section{Agradecimientos}

A mi querida familia.

\section{Bibliografía}

1. Muñiz-Albaiceta G. Curvas presión-volumen en la lesión pulmonar aguda. Med Intensiv. 2009;33:243-50.

2. Cole KS. Some physical aspects of bioelectric phenomena. Proc Natl Acad Sci USA. 1949;35:558-66.

3. Nopp P, Rapp E, Pfützner H, Nakesch H, Ruhsam CH. Dielectric properties of lung tissue as a function of air content. Phys Med Biol. 1993;38:699-716.

4. Kim Y, Webster JG, Tompkins WJ. Electrical impedance imaging of the thorax. J Microw Power. 1983;18:245-57.

5. Brown BH, Seagar AD. The Sheffield data collection system. Clin Phys Physiol Meas. 1987;8:91-7.

6. Casas O, Rosell J, Bragós R, Lozano A, Riu PJ. A parallel broadband real-time system for electrical impedance tomography. Physiol Meas. 1996;17:A1-6.

7. Breckon WR, Pidcock MK. Mathematical aspects of impedance imaging. Clin Phys Physiol Meas. 1987;8 Suppl A:77-84.

8. Breckon WR, Pidcock MK. Data errors and reconstruction algorithms in electrical impedance tomography. Clin Phys Physiol Meas. 1988;9 Suppl A:101-4.

9. Barber DC. A review of image reconstruction techniques for electrical impedance tomography. Med Phys. 1989;16:162-9.

10. Dobson DC, Santosa F. An image enhancement technique for electrical impedance tomography. Inverse Probability. 1994;10:317-34.

11. Mueller JL, Siltanen S, Isaacson D. A direct reconstruction algorithm for electrical impedance tomography. IEEE Trans Med Imaging. 2002;6:555-9.

12. Lionheart WR. EIT reconstruction algorithms: Pitfalls, challenges and recent developements. Physiol Meas. 2004;25:125-42.

13. Barber DC, Brown BH. Applied potential tomography. J Br Interplanet Soc. 1989;42:391-3.

14. Barber DC. Quantification in impedance imaging. Clin Phys Physiol Meas. 1990;11 Suppl A:45-6.

15. Harris ND, Suggett AJ, Barber DC, Brown BH. Application of applied potential tomography (AT) in respiratory medicine. Clin Phys Physiol Meas. 1987;8:155-65.

16. Adler A, Amyot R, Guardo R, Bates JH, Berthiaume Y. Monitoring changes in lung air and liquid volumes with electrical impedance tomography. J Appl Physiol. 1997;83:1762-7.

17. Balleza M, Fornos J, Calaf N, Feixas T, González M, Antón D, et al. Seguimiento del patrón ventilatorio en reposo mediante tomografía por impedancia eléctrica (TIE). Arch Bronconeumol. 2007;43:300-3.

18. Erlandsson K, Odenstedt $H$, Lundin S, Stenqvist O. Positive end-expiratory pressure optimization using electric impedance tomography in morbidly obese patients during laparoscopic gastric bypass surgery. Acta Anaesthesiol Scand. 2006;50:833-9.

19. Balleza M, Fornos J, Calaf N, Feixas T, González M, Antón D, et al. Measuring breathing pattern in patients with chronic obstructive pulmonary disease by electrical impedance tomography. Arch Bronconeumol. 2009;45:320-4.

20. Hahn G, Sipinkova I, Braisch F, Hellige G. Changes in the thoracic impedance distribution under different ventilatory conditions. Physiol Meas. 1995;16:A161-73.

21. Hinz J, Hahn G, Neumann P, Sydow M, Mohrenweiser $P$, Hellige G, et al. End-expiratory lung impedance change enables bedside monitoring of end-expiratory lung volumen change. Intensive Care Med. 2003;29:37-43.

22. Marquis F, Coulombe N, Costa R, Gagnon H, Guardo R, Skrobik Y. Electrical impedance tomography's correlation to lung volume is not influenced by anthropometric parameters. J Clin Monit Comput. 2006;20:201-7.

23. Wrigge $H$, Zinserling $J$, Muders T, Varelmann D, Günther $U$, von der Groeben $\mathrm{C}$, et al. Electrical impedance tomography 
compared with thoracic computed tomography during a slow inflation maneuver in experimental models of lung injury. Crit Care Med. 2008;36:903-9.

24. Frerichs I, Hinz J, Herrmann P, Weisser G, Hahn G, Dudykevych $\mathrm{T}$, et al. Detection of local lung air content by electrical impedance tomography compared with electron beam CT. J Appl Physiol. 2002;93:660-6.

25. Victorino JA, Borges JB, Okamoto VN, Matos GF, Tucci MR, Caramez MP, et al. Imbalances in regional lung ventilation: $A$ validation study on electrical impedance tomography. Am J Respir Crit Care Med. 2004;169:791-800.

26. Hinz J, Neumann P, Dudykkevych T, Andersson LG, Wrigge $H$. Regional ventilation by electrical impedance tomography: A comparison with ventilation scintigraphy in pigs. Chest. 2003;124:314-22.

27. Richard JC, Pouzot C, Gros A, Tourevieille C, Lebars D, Lavenne $\mathrm{F}$, et al. Electrical impedance tomography compared to positron emission tomography for the measurement of regional lung ventilation: an experimental study. Critical Care. 2009;13:R82.

28. Kunst PW, de Vries PM, Postmus PE, Bakker J. Evaluation of electrical impedance tomography in the measurement of PEEP. induced changes in lung volume. Chest. 1999;115:1102-6.

29. Hinz J, Moerer O, Neumann P, Dudykevych T, Hellige G, Quintel $M$. Effect of positive end-expiratory-pressure on regional ventilation in patients with acute lung injury evaluated by electrical impedance tomography. Eur J Anaesthesiol. 2005;22:817-25.

30. Barbas CS, de Matos GF, Pincelli MP, da Rosa Borges E, Antunes T, de Barros JM, et al. Mechanical ventilation in acute respiratory failure: Recruitment and high possitive end-expiratory pressure are necessary. Curr Opin Crit Care. 2005;11:18-28.

31. Meier T, Luepschen $H$, Karsten J, Leibecke T, Grossherr $M$, Gehring $\mathrm{H}$, et al. Assessment of regional lung recruitment and derecruitment during a PEEP trial based on electrical impedance tomography. Int Care Med. 2008;34:543-50.

32. Costa EL, Borges JB, Melo A, Suarez-Sipmann F, Toufen Jr C, Bohm SH, et al. Bedside estimation of recruitable alveolar collapse and hyperdistension by electrical impedance tomography. Int Care Med. 2009;35:1132-7.

33. Bikker IG, Leonhardt S, Reis Miranda D, Bakker J, Gommers D. Bedside measurement of changes in lung impedance to monitor alveolar ventilation in dependent and non-dependent parts by electrical impedance tomography during a positive endexpiratory pressure trial in mechanically ventilated intensive care unit patients. Crit Care. 2010;14:R100.

34. Zhao Z, Steinmann D, Frerichs I, Guttmann J, Möller K. PEEP titration guided by ventilation homogeneity: a feasibility study using electrical impedance tomography. Crit Care. 2010;14:R8.

35. Kunst PW, Bohm SH, Vazquez de Anda G, Amato MB, Lachmann B, Postmus PE, et al. Regional pressure volume curves by electrical impedance tomography in a model of acute lung injury. Crit Care Med. 2000;28:178-83.

36. Hinz J, Moerer O, Neumann P, Dudykevych T, Frerichs I, Hellige $\mathrm{G}$, et al. Regional pulmonary pressure volume curves in mechanically ventilated patients with acute respiratory failure measured by electrical impedance tomography. Acta Anaesthesiol Scand. 2006;50:331-9.

37. Lowhagen $\mathrm{K}$, Lindgren $\mathrm{S}$, Odenstedt $\mathrm{H}$, Stenqvist $\mathrm{O}$, Lundin $\mathrm{S}$. A new non-radiological method to assess potential lung recruitability: a pilot study in ALI patients. Acta Anaesthesiol Scand. 2011;55:165-74.

38. Kunst PW, Vazquez de Anda G, Bohm SH, Faes TJ, Lachmann B, Postmus PE, et al. Monitoring of recruitment and derecruitment by electrical impedance tomography in a model of acute lung injury. Crit Care Med. 2000;28:3891-5.

39. Lowhagen K, Lundin S, Stenqvist O. Regional intratidal gas distribution in acute lung injury and acute respiratory distress sindrome assessed by electric impedance tomography. Minerva Anestesiologica. 2010;76:1024-35.
40. Van Genderingen HR, van Vught AJ, Jansen JR. Regional lung volume during high-frequency oscillatory ventilation by electrical impedance tomography. Crit Care Med. 2004;32: 787-94.

41. Wolf GK, Grychtol B, Frerichs I, van Genderingen HR, Zurakowski D, Thompson JE, et al. Regional lung volume changes in children with acute respiratory distress syndrome during a derecruitment maneuver. Crit Care Med. 2007;35:1972-8.

42. Eyboglu BM, Brown BH, Barber DC. In vivo imaging of cardiac related impedance changes. IEEE Eng Med Biol Mag. 1989;8:39-45.

43. Brown BH, Barber DC, Morice AH, Leathard AD. Cardiac and respiratory related electrical impedance changes in the human thorax. IEEE Trans Biomed Eng. 1994;41:729-34.

44. Fagerberg A, Stenqvist O, Aneman A. Electrical impedance tomography applied to assess matching of pulmonary ventilation and perfusion in a porcine experimental model. Crit Care. 2009;13:R34.

45. Deibele JM, Luepschen H, Leonhardt S. Dynamic separation of pulmonary and cardiac changes in electrical impedance tomography. Physiol Meas. 2008;29:S1-14.

46. Brown BH, Leathard A, Sinton A, McArdle FJ, Smith RW, Barber DC. Blood flow imaging using electrical impedance tomography. Clin Phys Physiol Meas. 1992;13:175-9.

47. McArdle FJ, Suggett AJ, Brown BH, Barber DC. An assessment of dynamic images by applied potential tomography for monitoring pulmonary perfusion. Clin Phys Physiol Meas. 1988;9:87-91.

48. Smit HJ, Handoko ML, Vonk Noordegraaf A, Faes TJ, Postmus PE, de Vries PM, et al. Electrical impedance tomography to measure pulmonary perfusion: is the reproducibility high enough for clinical practice? Physiol Meas. 2003;24: 491-9.

49. Smit HJ, Vonk-Noordegraaf A, Marcus JT, van der Weijden S, Postmus PE, de Vries PM, et al. Pulmonary vascular responses to hypoxia and hyperoxia in healthy volunteers and COPD patients measured by electrical impedance tomography. Chest. 2003;123:1803-9.

50. Leathard AD, Brown BH, Campbell JH, Zhang F, Morice AH, Tayler D. A comparison of ventilatory and cardiac related changes in EIT images of normal human lungs and of lungs with pulmonary emboli. Phys Meas. 1994;15:137-46.

51. Kunst PW, vonk Noordegraaf A, Hoekstra OS, Postmus PE, de Vries PM. Ventilation and perfusion imaging by electrical impedance tomography: a comparison with radionuclide scanning. Physiol Meas. 1998;19:481-90.

52. Muders $\mathrm{T}$, Luepschen $\mathrm{H}$, Zinserling J, Meier T, Wrigge $\mathrm{H}$, Hedenstierna $G$, et al. Influence of different PEEP setting strategies on ventilation/perfusion matching in porcine ALI. Am J Respir Crit Care Med. 2009;179:A4673.

53. Noble TJ, Morice AH, Channer KS, Milnes P, Harris ND, Brown $\mathrm{BH}$. Monitoring patients with left ventricular failure by electrical impedance tomography. Eur J Heart Fail. 1999;1: 379-84.

54. Costa EL, Chaves CN, Gomes S, Beraldo MA, Volpe MS, Tucci MR, et al. Real-time detection of pneumothorax using electrical impedance tomography. Crit Care Med. 2008;36:1230-8.

55. Arad M, Zlochiver S, Davidson T, Shoenfeld Y, Adunsky A, Abboud $\mathrm{S}$. The detection of pleural effusion using a parametric EIT technique. Physiol Meas. 2009;30:421-8.

56. Steinmann D, Stahl CA, Minner J, Schumann S, Loop T, Kirschbaum A, et al. Electrical impedance tomography to confirm correct placement of double-lumen tube: a feasibility study. Br J Anaesth. 2008;101:411-8.

57. Serrano RE, Lema BD, Casas O, Feixas T, Calaf N, Camacho V, et al. Use of electrical impedance tomography (EIT) for the assessment of unilateral pulmonary function. Physiol Meas. 2002;23:211-20. 
58. Vonk-Noordegraaf A, Janse A, Marcus JT, Bronzwaer JG, Postmust PE, Faes TJ, et al. Determination of stroke volume by means of electrical impedance tomography. Physiol Meas. 2000;21:285-93.

59. Stout CL, Van de Water JM, Thompson WM, Bowers EW, Sheppard SW, Tewari AM, et al. Impedance cardiography: can it replace thermodilution and the pulmonary artery catheter? Am Surg. 2006;72:728-32.

60. Frerichs I, Dudykevych T, Hinz J, Bodenstein M, Hahn G, Hellige $G$. Gravity effects on regional lung ventilation determined by functional EIT during parabolic flights. J Appl Physiol. 2001;91:39-50.
61. Tobin $M$, Jenouri A, Lind B, Watson $H$, Schneider A, Sackner M. Validation of respiratory inductive plethysmography in patients with pulmonary disease. Chest. 1983;127: 615-20.

62. Kunst PW, Vonk Noordegraaf A, Straver B, Aarts RA, Tesselaar CD, Postmus PE, et al. Influences of lung parenchyma density and thoracic fluid on ventilatory EIT measurements. Physiol Meas. 1998;19:27-34.

63. Bikker IG, Leonhardt S, Bakker J, Gommers D. Lung volume calculated from electrical impedance tomography in ICU patients at different PEEP levels. Int Care Med. 2009;35: 1362-7. 